\title{
Listériose : la pointe de l'iceberg
}

Publié sur www.cmaj.ca le 19 septembre 2008.

$\mathrm{E}$ n août, le Canada a connu la pire épidémie de listériose au monde. Au 12 septembre 2008, des viandes froides contaminées provenant d'une usine de transformation des viandes de Toronto avaient fait au moins 16 victimes sur 43 cas confirmés de listériose dans la moitié des provinces. Le nombre de décès a déjà dépassé le double de celui de la fameuse éclosion d'Escherichia coli à Walkerton (Ontario). Et comme Listeria monocytogenes peut demeurer latente pendant deux à trois mois, il se peut que d'autres personnes meurent, deviennent malades ou subissent d'autres effets comme les fausses couches.

Mais que s'est-il donc passé? Comme dans le cas des épidémies de Walkerton et de SRAS, une éclosion de cette ampleur peut signaler des défaillances systémiques à de multiples niveaux. Listeria est l'agent biologique, les viandes froides sont le vecteur, mais il se pourrait que le problème remonte à des décisions gouvernementales risquées.

En novembre dernier, le gouvernement du Canada a entrepris un examen stratégique de l'Agence canadienne d'inspection des aliments (ACIA). L'examen a abouti notamment au transfert des fonctions d'inspection des viandes prêtes à manger des inspecteurs du gouvernement à l'industrie de la viande. Le Cabinet a décidé de «faire moins d'inspections sur place et davantage de surveillance, [ce qui] permettrait à l'industrie d'adopter des programmes de contrôle de la qualité et de gérer les principaux risques ${ }^{1} \gg$.

En pratique, la nouvelle politique signifiait que les agents de l'ACIA entreraient rarement dans des usines de transformation des viandes pour y effectuer des tests de dépistage de la présence de bactéries et qu'on s'en remettait surtout aux entreprises pour effectuer les tests. L'auto-inspection en est venue en grande partie à remplacer l'inspection gouvernementale au lieu de la compléter. Les mécanismes d'autoinspection ont donné des résultats efficaces dans d'autres pays, mais quelque chose a déraillé sérieusement au Canada. Il y a un signe troublant : même maintenant, des mois après le changement de politique, la procédure d'échantillonnage obligatoire de l'ACIA est toujours en développement.

Les Aliments Maple Leaf, l'entreprise dont une usine est à l'origine de la contamination par Listeria, a été une des premières à adopter le nouveau plan gouvernemental. Et pourquoi pas? La nouvelle politique facilitait l'auto-inspection : l'entreprise devait maintenir des bonnes pratiques de fabrication à son usine et vérifier les produits finis une fois par mois seulement ${ }^{2}$.

Le gouvernement du Canada a aussi accepté des normes nationales de contrôle de Listeria moins contraignantes que celles de bien d'autres pays. Santé Canada tolère la présence de 100 bactéries par gramme d'aliment prêt à manger au début de la vie du produit, même si la dose de Listeria ingérée en bout de ligne peut être plus importante parce que la bactérie peut se multiplier pendant la vie du produit, même s'il est réfrigéré 3 . À titre de comparaison, la Commission du Codex Alimentarius des Nations Unies et de l'Organisation mondiale de la Santé a accepté à contrecœur une norme tolérant la présence de 100 bactéries par gramme, mais seulement à la fin de la vie du produit ${ }^{4}$. Aux États-Unis, le gouvernement est encore plus sévère : c'est tolérance zéro bactérie ${ }^{5}$.

Confronté à la réalité de ces normes tolérantes sur Listeria, le gouvernement du Canada ne les a pas resserrées mais il a plutôt exercé des pressions pour que les États-Unis abaissent leurs normes ${ }^{2}$. L'ACIA s'est opposée particulièrement aux «visites quotidiennes» d'inspection et à «l'analyse du produit fini pour détecter la présence de Listeria», mesure de protection qui aurait pu repérer le problème de Listeria des Aliments Maple Leaf plus tôt et éviter ou atténuer l'impact de cette éclosion ${ }^{6}$.

Les erreurs stratégiques du gouvernement ont contribué à déclencher cette épidémie. Le gouvernement n'a pris aucune mesure corrective : il a seulement publié un rappel d'aliments, ce qui est étonnant. Les dirigeants vantent même la réussite de notre système de surveillance des maladies infectieuses - comme si, avec 16 décès, il y avait de quoi célébrer - tandis que les normes sur la salubrité des aliments demeurent aussi tolérantes que jamais.

L'épidémie de listériose rappelle à point nommé que le gouvernement Harper a balayé une grande partie des progrès réalisés par les gouvernements précédents en santé publique. Après l'épidémie de SRAS de 2003 et les recommandations subséquentes du Comité consultatif national sur le SRAS et la santé publique ${ }^{7}$ le gouvernement a créé l'Agence de la santé publique du Canada (ASPC) et l'a dotée de son propre ministre - lui donnant ainsi accès directement au premier ministre. En 2006, toutefois, une des premières mesures qu'a prises le premier ministre Stephen Harper a été d'éliminer le poste de ministre responsable de l'ASPC et par conséquent le siège de la santé publique à la table du Cabinet. Son gouvernement a aussi relégué le médecin hygiéniste en chef à la fonction publique, relevant du ministre de la Santé, laissant ainsi le pays sans porte-parole national indépendant sur les enjeux de la santé publique et sans leadership visible au cours de cette crise.

Il se pourrait toutefois que la listériose ne soit que la pointe de l'iceberg. La décision du Cabinet de novembre 2007 qui confiait l'auto-inspection aux propriétaires d'usines de transformation des viandes, faisait la même chose pour les exploitants de minoteries de provendes et réduisait en outre le programme de préparation à la grippe aviaire. Or, de mauvaises provendes sont à l'origine de l'épidémie d'encéphalite spongiforme bovine (maladie de la vache folle), et une pandémie de grippe pourrait faire des dizaines de milliers de 
victimes au Canada ${ }^{8}$. La listériose pâlit devant la comparaison. Dans l'ensemble, il semblerait que comme pays, le Canada est moins bien préparé à faire face aux épidémies maintenant qu'il l'était par le passé.

Pour s'attaquer au problème de santé publique de plus en plus grave un jour à peine avant de déclencher des élections, le premier ministre Harper a demandé la tenue d'une «enquête indépendante» sur l'épidémie de listériose. La structure de l'enquête proposée déçoit toutefois profondément. Selon le mandat de l'enquête, affiché sur le site web du premier ministre, on n'a pas choisi et on ne choisira pas un enquêteur indépendant du gouvernement. L'enquêteur ne pourra forcer des témoins à comparaître ni faire venir des documents. Le public ne pourra participer à l'enquête et on n'a pris aucun engagement de publier les constatations de l'enquêteur ou de faire rapport au Parlement'.

Une telle enquête ne sera pas à la hauteur de celles qui se sont tenues récemment au Canada : les enquêtes sur le scandale du sang contaminé, sur l'éclosion à Walkerton et sur l'épidémie de SRAS ont été des exercices indépendants convoqués par un commissaire indépendant (habituellement un juge) qui a tenu des audiences ouvertes et publiques et a été investi de tous les pouvoirs mentionnés ci-dessus. Dans les rapports publics de ces enquêtes antérieures, on a catalogué les défaillances institutionnelles à l'origine des épidémies et formulé des recommandations logiques sur la gouvernance des soins médicaux afin de mieux protéger les Canadiens. La commission d'enquête Krever sur le scandale du sang contaminé, par exemple, a légué un des meilleurs systèmes de collecte et de mise en banque de sang au monde ${ }^{10}$.

Le premier ministre Harper s'est déclaré «très troublé» par l'éclosion de Listeria. Nous le sommes aussi : Listeria est une bactérie omniprésente dans le sol. Il y aura inévitablement d'autres épidémies de listériose d'origine alimentaire. Une enquête publique complète sur les principales défaillances du système d'inspection des aliments du Canada s'impose pour protéger les Canadiens contre des menaces d'épidémies à venir. La population canadienne ne devrait accepter rien de moins. Il s'agira de la première étape et de la plus importante pour assainir notre chaîne alimentaire. Les politiciens feraient bien d'expliquer leur position au sujet d'une telle enquête avant le jour du scrutin.

\section{Amir Attaran LLB DPhil}

Chaire de recherche du Canada en droit, santé des populations et politique du développement mondial Université d'Ottawa Ottawa (Ont.)
Noni MacDonald MD MSc

Rédactrice de la section, Santé publique

Matthew B. Stanbrook MD PhD

Rédacteur adjoint, Sciences

Barbara Sibbald BJ

Rédactrice adjointe, Actualités et Humanités

Ken Flegel MDCM MSc

Rédacteur associé principal

Rajendra Kale MD

Rédacteur adjoint principal

Paul C. Hébert MD MHSc

Rédacteur en chef

$J A M C$

Intérêts concurrents: Voir www.cmaj.ca/misc/edboard.shtml.

Traduit par le Service de traduction de l'AMC.

\section{RÉFÉRENCES}

1. Conseil du Trésor. Décision du Conseil du Trésor - réunion du 13 novembre 2007. C.T. 834007. Ottawa (Ont.) : Le Conseil; 2008. Disponible [site web du Globe and Mail] : www.theglobeandmail.com/v5/content/pdf/cfiamemo.pdf (consulté le 12 septembre 2008).

2. Ha TT, Curry B, McIlroy A. Inspectors failed to adopt more rigorous US measures. Globe and Mail [Toronto] 27 août 2008. Disponible : www.theglobeandmail .com/servlet/story/RTGAM.20080827.wmeat27/BNStory/National/home (consulté le 12 septembre 2008).

3. Direction des aliments, Direction générale des produits de santé et des aliments, Santé Canada. Politique sur la présence de Listeria monocytogenes dans les aliments prêts à manger. Ottawa (Ont.) : Santé Canada; 2004. Disponible : www.hc-sc .gc.ca/fn-an/alt_formats/hpfb-dgpsa/pdf/legislation/policy_listeria_monocytogenes _politique_toc-fra.pdf (consulté le 3 septembre 2008)

4. Autorité européenne de sécurité des aliments. Request for updating the former SCVPH opinion on Listeria monocytogenes risk related to ready-to-eat foods and scientific advice on different levels of Listeria monocytogenes in ready-to-eat foods and the related risk for human illness. Scientific opinion of the Panel on Biological Hazards. EFSA Journal 2007;599:1-42. Disponible : www.efsa.europa.eu /cs/BlobServer/Scientific_Opinion/biohaz_op_ej599_listeria_en,3.pdf?ssbinary=true (consulté le 3 septembre 2008).

5. États-Unis DHHS Food and Drug Administration's Center for Food Safety and Applied Nutrition (FDA/CFSAN). Quantitative assessment of relative risk to santé publique from foodborne Listeria monocytogenes among selected categories of ready-to-eat foods. Rockville (MD) : The Administration; 2003. Disponible : www .foodsafety.gov/ dms/lmr2-1.html (consulté le 12 septembre 2008).

6. Curry B. Ottawa wanted US to accept more lenient meat inspection regime. Globe and Mail [Toronto] 12 août 2008.

7. Santé Canada. Leçons de la crise du SRAS : Renouvellement de la santé publique au Canada : un rapport du Comité consultatif national sur le SRAS et la santé publique. Octobre 2003. Ottawa (Ont.) : Agence de la santé publique du Canada; 2003. Disponible : www.phac-aspc.gc.ca/publicat/sars-sras/pdf/sras-f.pdf (consulté le 12 septembre 2008).

8. Agence de la santé publique du Canada. Plan canadien de lutte contre la pandémie d'influenza dans le secteur de la santé. Ottawa (Ont.) : L'Agence; 2006. Disponible www.phac-aspc.gc.ca/cpip-pclcpi/s02-fra.php\#23 (consulté le 12 septembre 2008).

9. Le premier ministre annonce le mandat d'une enquête indépendante sur l'éclosion de listériose [communiqué]. Ottawa (Ont.) : Bureau du premier ministre du Canada; 6 septembre 2008. Disponible : http://pm.gc.ca/fra/media.asp?category $=1 \& i d=2268$.

10. Wilson K. The Krever Commission - 10 years later. JAMC 2007;177:1387-9. 